\title{
Ending the Karl Pearson controversy (1904): Over the incomplete couplets produced by Mendel's fraction-addition method
}

\author{
Conrado Ruiz-Hernández
}

Departments of History of Biology and Environmental Education at the National Autonomous University of Mexico: UNAM-Iztacala, Los Reyes Iztacala, Tlalnepantla-Estado de México, México. 54090

\author{
Email address \\ cruiz@campus.iztacala.unam.mx (C. Ruiz-Hernández)
}

\section{To cite this article:}

Conrado Ruiz-Hernández. Ending the Karl Pearson Controversy (1904): Over the Incomplete Couplets Produced by Mendel's FractionAddition Method. International Journal of Genetics and Genomics. Vol. 2, No. 6, 2014, pp. 121-125.

doi: 10.11648/j.ijgg.20140206.14

\begin{abstract}
Early in the 20th Century, leading mathematicians found a link between Mendel's Laws and Newton's Binomial. This enabled multigenerational studies of entire populations. In this regard, K. Pearson in 1904 raised objections to Mendel's predictions that the 'pure' (dominant and recessive) descendants of hybrid ancestors turn out to be incomplete assemblies when using the sum of fractions used by Mendel in his 1866 article "Experiments in Plant Hybridization". This algorithm is analyzed as a model for the case of just one hereditary characteristic, within an axiomatic framework that necessitates the formulation of a theorem in order to elucidate whether it was, on the one hand, a genuine mistake or, on the other, it is what Mendel, with all conviction and consideration, intended to say. We take into account the contemporary (1850-1870) knowledge of the cell and the structures involved in the transmission of inherited characteristics that this pioneer in the field of genetics would have had available for his deliberations at a time when this discipline was not yet a science. There follows the analysis of an unspecified intermediate member of the sum of fractions (not included in the Mendel's original paper), which, from a mathematical standpoint, helps us resolve the incomplete assemblies ('pure' descendants) enigma.
\end{abstract}

Keywords: Mendel's Laws, Transmission of Inherited Characteristics, Mendel's Fraction-Addition Method, Hardy- Weinberg Law, and Newton's Binomial

\section{Introduction}

When, in about 1850, Johann Gregor Mendel began his methodical study of the transmission of inherited characteristics, focusing mainly on a variety of domestic pea (Pisum sativum), Biology was going through a stage of intense consolidation as a science: Cell theory was being consolidated, animal physiology was in the process of becoming an experimental field and Charles Darwin and Alfred Russell Wallace were working hard to organize their ideas about evolution [1]. In other fields of biology, the endeavor of delving more deeply into knowledge already known was generating great intellectual excitement, especially the largely un-deciphered mystery of what was contained inside the cell (already known as the basic unit of living organisms), and the cell structures which at that time existed as mere suspicions [3]. This is the backdrop to Mendel's successful attempt to understand the mechanisms that govern the transmission of inherited characteristics in an age when the existence of genes was still unknown [2, 21, $22]$. How could this happen? He combined an exquisite and extensive body of experimental work with his commanding grasp of mathematical reasoning. Being a teacher of horticulture and higher level mathematics were part of Mendel's duties as a Catholic priest [18].

The axiomatic method is an intellectual process designed to test facts, suppositions and conjectures that were hitherto considered to be self-evident. This foments the verification and credibility of several fields of knowledge, particularly in areas where, for the most part, the available information is precarious and elements of proof are not readily available. It may have been founded by followers of Thales of Milletus in Ionia around the VI century B.C. Thales of Milletus was considered to be a forerunner of Greek science and philosophy [9]. Self-evident truths are considered to be of two kinds: ones which don't require proof (axioms) and those that with few exceptions do need to be proved 
(theorems). The building of scientific knowledge is based on the latter. Hypotheses in the modern experimental method are in a way more like theorems. When applied to mathematical matters, this method is usually very rigorous, because it was at one time related to the ideal of deductive systematization whose first principles were one of Aristotle's contributions to an explanation of facts pertaining to a mostly divinely-conceived material reality. During the XVIIth century, Newton reformulated this method by his clever use of supporting mathematics. He saw it as a useful resource for the understanding of physical facts, taking into account that the mathematical description could only approach reality (in some cases more, in some cases less), without the need for this reality to be subject to numbers and equations. In other words, in this vision, mathematics is considered to be a means rather than an end [13].

Early in the 20th Century, leading mathematicians found a link between Mendel's Laws and Newton's Binomial. This enabled multigenerational studies of entire populations [6, 19 , 24]. Some historians have also used the Newton binomial: (a $+\mathrm{b})^{2}=\mathrm{a}^{2}+2 \mathrm{ab}+\mathrm{b}^{2}$, in order to explain Mendel's second law $[11,12,14]$. However, these developments took place later than the work performed by the pioneer of genetics.

Synopsis of Mendel's Laws

The principles or laws (according to followers of Mendelian ideas) in the first two decades of the past century $[10,16,23]$, were formulated by Mendel in 1866 [15] They predict the expected descendants when two organisms are crossed for reproductive purposes: firstly, when they have pure features of one single characteristic, or, secondly, when they have hybrid characteristics either in the case of both parents having the dominant (always expressed) or the recessive characteristic (expressed only when both allele or chromosome parts are carriers of the same feature). As a result of the experiments he performed, he synthesized his findings in the following mathematical expression (pages 16 and 24 of his first article).

$$
\mathrm{A} / \mathrm{A}+\mathrm{A} / \mathrm{a}+\mathrm{a} / \mathrm{A}+\mathrm{a} / \mathrm{a}=\mathrm{A}+2 \mathrm{Aa}+\mathrm{a} .
$$

The dominant characteristic is expressed with a capital letter and the recessive characteristic is expressed with a lower case letter. According to the taxonomic system he invented, for every inheritable characteristic or feature, a different letter is given. We must say that Mendel could only watch forms easily seen ("A", "a" and "Aa"), but not invisible cell structures that he didn't know about. Nevertheless, in spite of these limitations, he did possess an incipient notion of the theories of the cell and of fertilization. An example of this was his idea that the inherited characteristics of parents would unite, fuse or somehow join together in the offspring. When two pure organisms are crossed, whether the characteristic is dominant or recessive, the resultant offspring will have the same characteristic of parents: $\mathrm{AA}=\mathrm{A}$ and $\mathrm{aa}=\mathrm{a}$, because one $\mathrm{X}$ one $=$ one. The associated mathematical reduction is formal and not structural. But, genetically, we have: $\mathrm{AA}=\mathrm{A}+\mathrm{A}$ and $\mathrm{aa}=\mathrm{a}$ $+a$, by the aggregation that occurs between the hereditary traits of the offspring.

His First Law (uniform assortment of two or more traits from gamete permutation) establishes the fact that all hybrid organisms provide both dominant and recessive pure lines, they are equal: $\mathrm{AA} x$ aa $=\mathrm{Aa}+\mathrm{Aa}+\mathrm{aA}+\mathrm{aA}$. The second law (segregation assortment of two or more traits) establishes that when two hybrid organisms are crossed (Aa x aA), the offspring will have the following proportions: 1 (pure dominant organism): 2 (hybrid organisms): 1 (pure recessive organism) or 3 (pure dominant and hybrids):1. In the case of real data, we consider a $5 \%$ variation, where the proportion must be nearly $(3 \pm 0.15)$ to 1 to be a very good result. It should be noted that Mendel reported in the seven experiments performed, ratios that are on an individual basis within the range indicated. The unit (1) can only pertain to a pure recessive individual. This is useful as a pattern to calculate other proportions. Mendel reported that for his experiments overall, there was an average proportion of 2.98 to 1 . This has provoked serious criticism because it makes one think he might have altered or forged his field data $[5,8]$, because achieving an accurate proportion $1: 2: 1$ or 3 to 1 , using real field data, is statistically impossible. In other words, it would, genetically speaking, be like squaring the circle.

Finally, the third law of inheritance (independent assortment of two or more traits) predicts that possible combinations of several characteristics are independent and they may be mixed in every possible way - that is, $3^{\mathrm{n}}$.

Objectives

1 Review the mathematical approaches that Mendel explained in his first article.

2 Analyze the mathematical development of the second law of heredity, which in fact synthesizes Mendel's approaches.

3 Break down Mendel's Fraction-Addition Method, with the aim of understanding the mathematical details involved, as well as the simplification criteria he applied to get his result.

4 Clarify whether the pure descent foreseen by Mendel is full or incomplete assembly, as this constitutes $\mathrm{K}$. Pearson's main criticism of Mendel's work.

\section{Method}

1 Embody Mendel's mathematical approaches in an axiomatic framework. To do this, we must establish a theorem.

2 Mathematical analysis.

3 Review the literature in which the authors criticize the purely mathematical claims of Mendel's work. Those which are not purely statistical or stemming from the way he obtained his field data.

Hypothesis

"Correct application of the fraction-addition method that Mendel used to describe his heredity predictions, coupled with the axiomatic position he makes explicit in his first article, enabled him to arrive at a theoretical result that was mathematically correct".

Analysis of Fraction Addition 
Mendel uses the following scheme to establish possible combinations in hybrid descent. (He places the pollen cells on the higher row and the germinal cells on the lower row):

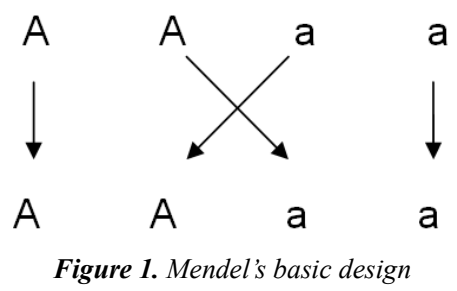

However, the combination scheme requires further explanation. Mid XIXth century farmers in those countries that had developed modern agricultural practices designed animal and plant crosses using in their reproductive plans rudimentary prototypes of double entrance squares [4, 25]. Mendel's empirical model was organized and designed in this manner:

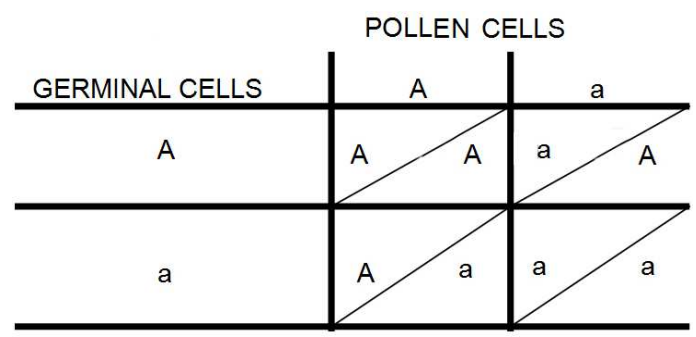

Figure 2. Mendel's combinatory analysis

He believed that for every two gametes one carries the dominant trait and other the recessive. Once combinations have been thus assembled, with the male gamete as the numerator and the female as denominator, Mendel deduces the theoretical proportion of the hybrid parents' offspring.

Now, while solving the fraction-addition, we obtain another element (shaded gray):

$$
\frac{A}{A}+\frac{A}{a}+\frac{a}{A}+\frac{a}{a}=\frac{A a+A A+a a+a A}{A a^{*}}=A^{+} 2 A a^{+} a .
$$

Where we consider Mendel's assertion regarding fractions (which by extension we apply to products and literals) as distinct biological units -individual organisms- functioning as a theorem (a self-evident truth that must be proved), then we have:

$\mathrm{AA}=\mathrm{A}, \mathrm{aa}=\mathrm{a}, \mathrm{Aa}+\mathrm{aA}=2 \mathrm{Aa}$ (where the well-known 'factor order does not alter product' principle applies), and the lowest common denominator ( $\mathrm{Aa}^{*}$ ) he reduces to the unit (1). Term-by-term deductive simplification is applied to the sum of products; although this same we can do it directly on the sum of fractions [20]. Even though Mendel didn't include in his original approach (p. 29-30) the shaded area (where the pure offspring do appear in double letters: AA and aa), we may think that in order to avoid confusion he would have done so.

Can we observe the same thing when speaking about Newton's binomial?

Newton binomial's application to the hybrid parents expected offspring comes from the "Aa" hybrid separation in the $(\mathrm{A}+\mathrm{a})$ or $(\mathrm{a}+\mathrm{A})$ binomial, considering the dominant and recessive permutations. The multiplying or crossing of both binomials gives the following result: $(A+a) \times(a+A)=$ $\mathrm{A}+2 \mathrm{Aa}+\mathrm{a}$, which is the same as with the fraction-addition result. However, Mendel was only able to observe surfaceappearance forms ("A", "a" and "Aa") and not structures that could be separated from the heterozygote allele in: $\left(A+A^{\prime}\right)$, $\left(\mathrm{a}+\mathrm{a}^{\prime}\right),\left(\mathrm{A}+\mathrm{a}^{\prime}\right)$ and $\left(\mathrm{a}+\mathrm{A}^{\prime}\right)$. Mendel couldn't make this assertion because he was unaware of the existence of chromosomes as well as the allele which equally package the inherited characteristics. This involuntary ignorance of Mendel is ruthlessly criticized by Karl Pearson, suggesting the possible mathematical incompetence of the former (Pearson 1904, 505). [19]. Prime character of the pure organisms' second allele is to underline the heterozygote allele in these descendants just like in the case of hybrids. In the case of Mendel, the product "Aa" is just a taxonomic form (just like in the case of pure organisms " $A$ " and "a"), because he didn't know the real genetic structure of hybrid and pure descent. Robert Olby, in his influential 1979 article (p. 70), unfortunately omits this detail because he considers that the omission of the second allele in the 'pure' offspring (AA and aa) shows that Mendel has no notion whatsoever of particulate inheritance, believing only that elements or factors of the same kind would merge [17]. He fails to recognize that Mendel was correct in that a fraction of the male gamete (pollen cell) joins, in Mendel's words, 'traits united through fertilization', (p. 29) with another fraction of the feminine gamete (germinal cell). Mendel's understanding of this was in spite of the fact that the forerunner of genetics was unaware of the content of gametes nor, of course, that these were haploids. Purely by means of mathematical inference, Mendel was able to envision the abstract notion (nonmaterial or non-tangible, perhaps 'spiritual') of the as yet undiscovered gene. It appears in the fractions of all traits, albeit in a vision that is taxonomic-inheritance rather than cytological-genetic. This latter point is now a part of the ongoing debate regarding the analysis of Mendel's work [7]. The following commentary is still relevant: 'Each generation, perhaps, found in Mendel's paper only what it expected to find; in the first period a repetition of the hybridization results commonly reported, in the second a discovery in inheritance supposedly difficult to reconcile with continuous evolution. Each generation, therefore, ignored what did not confirm its own expectations' (Fisher 1936, 171). [8].

\section{Discussion}

Mendel's calculi origin, performed to establish the fractionaddition methodology, is the basis for his predictions about the offspring of hybrid parents. It is very close to the use of a double entrance combination table (in turn very similar to a Punnet square) and we see that, in this manner, the theoretical descent prediction was very close to his experimental results. The correspondence found between the result of fractionaddition and factorizing a binomial is an intrinsically 
mathematical coincidence, even though what has been observed in both cases is not exactly the same. Fractionaddition can be observed in appearance forms: "A", "a" and "Aa"; with the Newton binomial we need to distinguish the allele coming from different gametes (reproductive cells): (A+ $\left.\mathrm{a}^{\prime}\right),\left(\mathrm{a}+\mathrm{A}^{\prime}\right),\left(\mathrm{A}+\mathrm{A}^{\prime}\right)$ and $(\mathrm{a}+\mathrm{a})$. Mendel couldn't have had knowledge of this. Even though the reduction of $\mathrm{AA}=\mathrm{A}$ and $\mathrm{aa}=\mathrm{a}$ (see Appendix), is mathematically accurate, it is not exactly the same when we consider the specimen's morphology and appearance and the genetic structure of pure and hybrid descent. Mendel's mathematical reasoning shows a clear trend to abstraction; it is, therefore, highly probable that the laws of heredity were deduced by inference. However, verification of the offspring's real characteristics could only come from experimental work, mainly when the expected proportion for hybrid parents' offspring, 1:2:1, are obtained in the first generation, with three individuals showing "dominant" characteristics (where we have two masked hybrids), identifiable in the next generation by a self-cross and a recessive-only individual (the last necessarily "pure" individual). Experimental testing is a requirement of Newton's axiomatic method.

Certainly, Mendel was an expert gardener and a clever mathematician. Close as we are now to the hundred and fiftieth anniversary of the publication of Mendel's first article in 1866, and given all the available evidence, it is inadmissible to cast doubt on Mendel's mathematical wisdom by suggesting, as did K. Pearson (1904) and followers, that the result of the sum of fractions ( $A$ $+2 A a+a)$ is an incorrect result. A brief explanatory exercise: if $a=1$ and $b$ $=1,(a+b)^{2}=$ ? Regarding the product of " $a b$ ", " $a$ " and " $b$ " are the same units, so 1 × $1=1$. The only correct answer supported by Mendel's axiomatic platform is: $a+2 a b+b$ or four different individuals (4). On this point, we must recognize Mendel's truly ingenious final solution - to conserve the " $a$ " and " $b$ " terms in the solution as well as the product "ab", with the respective coefficients, since the numerical simplification (simply: $1+2+1$ ), would have little utility in the genetic sense. Nevertheless, applying K. Pearson's criteria to this same problem, the equally correct result is something else: $a^{2}+2 a b+b^{2}$ (or, as before, four different individuals). The difference lies in that in the first case, a taxonomy-heredity (Mendelian) criterion is applied, whereas in the second a cytology-genetics (K. Pearson) criterion is used. In conclusion, and in brief, we can simply affirm that from a strictly mathematical standpoint, it must be the case that: $A+2 A a+a=A A+2 A a+a a$.

\section{Conclusion}

The summing of fractions, especially the shaded element which is developed as an original contribution of this report - in the aforementioned equation, provided Mendel with all he needed for a correct solution of the relevant algorithm, completely embedded in an axiomatic framework. It is confirmed that the partial assemblies (homozygous) of the "pure" offspring (dominant and recessive) resulting from Mendel's fraction-addition are by no means genetically incomplete specimens, but are rather taxonomically apparent forms. Even though the coincidence of fraction-addition and the applying of this binomial is mathematically explicable, it is merely incidental and more appropriate when Man gained knowledge of the genetic and cell structures discovered and verified towards the end of the XIXth century. Chromosomes were discovered by Walther Flemming, a German cytologist, in 1882, two years before Mendel's passing away.

\section{The Axiomatic Basis of Mendel's Laws}

Equation described by Mendel in 1866 to calculate the expected offspring of hybrid parents (dominant trait in capital letters and recessive trait in lower case letter)

$$
\frac{A}{A}+\frac{A}{a}+\frac{a}{A}+\frac{a}{a}=A+2 A a+a \text {. }
$$

\section{Theorem}

"The fractions are distinct biological units".*

So, by extension, that products and literals are also unitary (individual organisms).

\section{AXIOMATIC ARGUMENT}

Definitions:

$\underline{A}=A A=A=I^{* *}$

A

$\underline{a}=a a=a=1 * *$

$\bar{a}$

Similarly, for hybrids:

$\underline{A}+\underline{a}=A a+a A=2 A a=2$

a $A$

The origin of hybrids:

$A A x a a=2 A a+2 a A^{* * *}$

Additional question: $* * * *$

$A a=A+a$ ?

Verification Using Newton's Axiomatic Method*****

Solving by binomial factorization (this procedure It was applied by $\mathrm{K}$ Pearson, but he believed to obtain a different result):

$$
(\mathrm{A}+\mathrm{a})^{2}=\mathrm{A}+2 \mathrm{Aa}+\mathrm{a} * * * * * *
$$

'Quot erat demonstrandum'

*Mendel establishes specific attributes for the mathematical description and symbols of his approaches. If this is to be verified, we need to assign them a theorem category (self-evident truth that requires verification).

**Mendel's reduction to units of all pure offspring from crossing dominant (AA) and recessive (aa) individuals. This is not a paradox: the literals are indivisible mathematical abstractions but with particular taxonomic and genetic characteristics, really 'sui generis'.

***Mathematical representation of Mendel's First Law.

$* * * *$ We don't have an evidence of this formulation by Mendel.

*****By using the axiomatic method in this manner, starting from written mathematical reasoning, we must accept that the observed phenomenon's real behavior is close to the established ideal. This is the opposite of the deductive systematization (primary geometric application) and Aristotle's orthodox principles.

******Mathematical representation of Mendel's Second Law. From it, we deduce Mendel's Third Law of (independent assortments according to characteristics seen: $\left.3^{n}\right)$; the calculation of total hybrid organisms $\left(2^{n}\right)$ as well as the minimum sample according to the design agreement $\left(4^{n}\right)$.

Figure 3. (Appendix):

\section{References}

[1] Beddall BG: Wallace, Darwin and the Theory of Natural Selection. A study in the Development of Ideas and Attitudes: Journal of the History of Biology, 1968, 1: 261-324.

[2] Bowler PJ: The Mendelian Revolution. The John Hopkins University Press, Baltimore 1989.

[3] Coleman W: Biology in the Nineteenth Century, Problems of Form, Function, and Transformation, Cambridge University Press, New York 1977. 
[4] Corcos A, Monaghan F: Gregor Mendel's experiments on plant hybrids: a guided study, New Brunswick: Rutgers University Press 1993.

[5] Di Trocchio F: Mendel's experiments: a reinterpretation, Journal of the History of Biology 1991, 24: 485-519.

[6] Edwards AW: G.H. Hardy (1908) and Hardy-Weinberg Equilibrium, Genetics 2008, 179: 1143-1150.

[7] Franklin A: The Mendel-Fisher controversy: an overview. In: Ending the Mendel-Fisher Controversy (Allan Franklin et al, Editors), University of Pittsburgh Press, Pittsburgh (PA) 2008, p. 1-77.

[8] Fisher RA: Has Mendel's work been rediscovered? Annals of Science 1936, 1: 115-137.

[9] Hull LW: History and Philosophy of Science: An Introduction. Longmans, London 1959.

[10] Hartl D, Orel V: What Gregor Mendel Think He Discovered? Genetics 1992, 131: 245-253.

[11] Iltis H: Gregor Johann Mendel, Springer Verlag, Berlin 1924.

[12] Ledesma-Mateos I: Historia de la biología, AGT Editor, Distrito Federal (México) 2000.

[13] Losee J: (Fourth Edition), A Historical Introduction to the Philosophy of Science, Oxford University Press, New York 2001, p. 72-85.

[14] Mark H: Gregor Johann Mendel on Pisum sativum: Archives of Ophthalmology 1966, 75: 287-289.

[15] Mendel G.: Experiments in Plant Hybridization (1866). In: Stern, C., and E. Sherwood, The Origin of Genetics: A Mendel Source Book. W. H. Freeman, San Francisco 1966, p. $16,24,29-30$.
[16] Olby RC: Mendel no Mendelian? History of Science 17: $53-$ 72. Reprinted with minor changes in The Origins of Mendelism, 2nd ed., 1979, pp.234-258. Chicago: Chicago University Press, 1985.

[17] Olby R: Mendel, mendelism and genetics. This essay appears, exclusively at Mendel Web 1997.

[18] Orel V: Gregor Mendel: the first geneticist, Oxford University Press, Oxford 1996.

[19] Pearson K: Mathematical contributions to the theory of evolution. XII. On a generalized theory of alternative inheritance, with special reference to Mendel's laws, Philosophical Transactions of the Royal Society 1904, 72: 505-509.

[20] Ruiz HC: Corrección a una equivocación de Karl Pearson (1904): la suma de fracciones de Mendel no produce acoplamientos genéticos incompletos. Ciencia ergo sum, 2014, 21(1): $67-70$

[21] Sandler I: Development: Mendel's Legacy to Genetics. Genetics 2000, 154: 7-11.

[22] Schwartz J: In pursuit of the gene: from Darwin to DNA, Harvard University Press, Cambridge (Massachusetts) 2008.

[23] Sturtevant A: (Republished Edition), History of genetics, Cold Spring Harbor Laboratory Press, @,Foundations of Classical Genetics 2001.

[24] Wilczynski J: Contributions to the theory and evolution of mendelian generalizations. Acta Biotheoretica, 1942, 6: 97152.

[25] Wood R, Orel V: Genetic prehistory in selective breeding: a prelude to Mendel, Oxford: Oxford University Press 2001. 